\title{
Diabetes mellitus y su impacto en la etiopatogenia de la sepsis
}

\author{
Limberth Machado-Villarroel, ${ }^{1}$ Mabel Montano-Candia, ${ }^{2}$ Diamanti Abraham Dimakis-Ramírez ${ }^{3}$
}

\section{Resumen}

Ciertas enfermedades infecciosas tienen mayor frecuencia y severidad en pacientes con diabetes mellitus (DM), lo cual incrementa sustancialmente las tasas de morbimortalidad. La DM incrementa el riesgo para bacteremia nosocomial adquirida en la comunidad. La mayor incidencia de infecciones en diabéticos está ocasionada por la hiperglucemia concomitante, lo cual ocasiona una disfunción inmunológica caracterizada por alteraciones de la función de los neutrófilos, actividad antioxidante e inmunidad humoral disminuida. Otras complicaciones son micro y macroangiopatía, neuropatía, trastornos en la motilidad urinaria y gastrointestinal, los cuales en conjunto contribuyen a la patogenia de los procesos infecciosos en estos pacientes. Actualmente se piensa que el involucro de productos finales de la glucosilación avanzada está asociado a inactivación de componentes inmunitarios, contribuyendo a una mayor vulnerabilidad frente a infecciones. Se ha propuesto una asociación entre hiperglucemia e infección en pacientes en estado crítico; no obstante, la evidencia para ello aún es limitada por la complejidad de los factores que intervienen. Distintos factores de riesgo influyen de forma negativa en el pronóstico de la sepsis severa tales como edad avanzada, inmunosupresión y alcoholismo crónico. Aún existe información contradictoria respecto al impacto que pudiese ejercer la DM en el pronóstico de pacientes sépticos.

Palabras clave: Choque séptico, diabetes mellitus 1, hiperglucemia, infección, inmunosupresión, sepsis.

\begin{abstract}
Summary
Certain infectious diseases have a higher frequency and severity in patients with diabetes mellitus (DM), which substantially increases morbidity and mortality rates. DM acts as a predisposing factor for bacteremia seen in both the nosocomial and community settings. The greater incidence of infections in diabetics is caused by the accompanying hyperglycemia, which generate an immune dysfunction characterized by disorders of the neutrophilic granulocytes, diminished antioxidant activity and humoral immunity. Other complications of DM such as micro or macroangiopathy, neuropathy, urinary and gastrointestinal motility disorders, among others, contribute to the pathogenesis of infectious processes in these patients. It is thought that cross-linking of advanced glycation end products is associated with inactivation of immune components, contributing to a higher vulnerability to infections. An association between hyperglycemia and infection in critically ill patients has been proposed; however, evidence remains limited. Several risk factors influence negatively in the prognosis of severe sepsis, such as advanced age, immunosuppression and chronic alcoholism. There still is contradictory data concerning the impact that DM could exert on the prognosis of septic patients.
\end{abstract}

Key words: Septic shock, 1 diabetes mellitus, hyperglycemia, infection, immunosuppression, sepsis.

\footnotetext{
${ }^{1}$ Especialista en Medicina Crítica y Cardioneumología. Universidad Autónoma de Ciudad Juárez (UACJ). Hospital Ángeles Ciudad Juárez.

2 Especialista en Nutriología Clínica y Pediatría HGZ Núm. 6 del IMSS, Ciudad Juárez.

3 Médico Pasante de Servicio Social de la Universidad Autónoma de Ciudad Juárez (UACJ).
}

Correspondencia:

Dr. Limberth Machado Villarroel

Hospital Ángeles Ciudad Juárez

Av. Campos Elíseos Núm. 9371, Fracc. Campos Elíseos, 32420, Cd. Juárez, Chihuahua, México.

Correo electrónico: limberthmv@hotmail.com

Aceptado: 04-05-2017.

Este artículo puede ser consultado en versión completa en http://www.medigraphic.com/actamedica 


\section{ANTECEDENTES}

En el año 2012 la diabetes mellitus (DM) fue responsable de 1.5 millones de defunciones a nivel global y se posicionó como la octava causa de muerte en ambos sexos, mientras que un total de 2.2 millones de muertes estuvieron asociadas a enfermedades cardiovasculares, nefropatía crónica y tuberculosis, relacionadas con niveles de glucemia superiores a las cifras óptimas. ${ }^{1,2}$ De acuerdo a reportes oficiales de la Organización Mundial de la Salud, en el 2014 había 422 millones de personas > 18 años de edad con DM en todo el mundo; $8.3 \%$ de los casos se ubicaban en el continente americano. La DM es una patología que afecta al $10 \%$ de la población general y está asociada a tasas elevadas de morbimortalidad, las cuales anticipan un incremento sustancial en las próximas décadas. ${ }^{3,4}$ Se estima que los pacientes con DM representan 13$26 \%$ de los ingresos hospitalarios; por tanto, imponen una considerable carga a los sistemas de salud a nivel global. ${ }^{5}$ Entre las principales complicaciones de la DM se encuentran la sepsis e insuficiencia renal, siendo la primera particularmente severa y responsable de aproximadamente $22 \%$ de las defunciones en dicho grupo de pacientes. ${ }^{6}$ Cabe mencionar que se hace alusión a la DM como entidad nosológica, ya que no existen estudios suficientes para efectuar una distinción entre DM tipos 1 y 2 (DM1 y DM2) en la etiopatogenia de la sepsis.

Sepsis es un término indicativo de un escenario clínico en el cual existe infección subyacente (confirmada y/o sospechada) así como síndrome de respuesta inflamatoria sistémica (SRIS) que incluye signos como fiebre o hipotermia, taquicardia, taquipnea, leucocitosis o leucopenia, no atribuibles a otras causas. ${ }^{7,8}$ Se sabe que la sepsis puede originarse en respuesta a múltiples causas infecciosas y que, además, la septicemia no constituye una condición invariable de la sepsis. Por otro lado, la sepsis severa representa un grado mayor de disfunción orgánica aguda (DOA) generalmente asociado a insuficiencia multiorgánica, la cual implica una carga de morbimortalidad superior a la sepsis sin DOA, lo cual da lugar a una utilización de casi el 50\% de los recursos en las Unidades de Cuidados Intensivos ( $\mathrm{UCl}){ }^{9}$ Se estima que en los Estados Unidos de América se presentan 750,000 nuevos casos de sepsis severa anualmente, constituyendo alrededor del $2 \%$ de los ingresos hospitalarios; asimismo representa casi el 10\% de los ingresos a la $\mathrm{UCl} .{ }^{10}$ Resulta necesario señalar el hecho de que diversas causas de origen infeccioso y no infeccioso son capaces de generar un SRIS, lo cual dificulta la diferenciación entre sepsis y otras condiciones patológicas con un cuadro clínico similar; por ello es común emplear de forma intercambiable los términos sepsis y sepsis severa, haciendo referencia a aquel síndrome infeccioso complicado por DOA. ${ }^{10}$ El choque séptico representa un cuadro clínico de sepsis complicado con hipotensión (la cual no responde a fluidoterapia); además cursa con hiperlactacidemia. La sepsis severa resulta de procesos infecciosos adquiridos en la comunidad o asociados a la atención de la salud, con la neumonía representando la principal causa (cerca del $50 \%$ de los casos), seguida de infecciones intraabdominales y de vías urinarias. ${ }^{11-13}$ Aunque usualmente es ocasionada por infecciones estafilocócicas o estreptocócicas, la sepsis también puede ser ocasionada por otras bacterias, hongos, virus y parásitos. $^{8,14}$ Las bacterias gram-positivas aisladas con mayor frecuencia son Staphylococcus aureus y Streptococcus pneumoniae, mientras que los organismos gram-negativos más representativos son Escherichia coli, Klebsiella spp. y Pseudomonas aeruginosa. ${ }^{15}$ Los factores de riesgo para sepsis actualmente reconocidos incluyen: edad (poblaciones pediátrica y geriátrica), sexo masculino, ascendencia africana u otra raza no caucásica, composición genética del huésped, función orgánica preexistente, empleo de fármacos inmunosupresores, así como la presencia de comorbilidades crónicas tales como cáncer, síndrome de inmunodeficiencia adquirida (SIDA) y enfermedad pulmonar obstructiva crónica (EPOC), entre otras. ${ }^{8}$

La DM actúa como factor predisponente para sepsis, tanto en el escenario intrahospitalario como a nivel de la comunidad. ${ }^{16}$ A nivel global, la tasa de mortalidad por sepsis severa fluctúa desde un 28.6-49.6\%..$^{17}$ A pesar de los avances relevantes en términos diagnósticos y terapéuticos, la tasa de supervivencia general en sepsis no ha sufrido modificaciones significativas en el transcurso del tiempo. ${ }^{18}$ La DM representa una de las principales comorbilidades con mayor impacto en pacientes sépticos en función de su elevada prevalencia y manifestaciones fisiopatológicas concomitantes. ${ }^{19}$ Entre los pacientes con sepsis severa, se reporta que alrededor del $10-30 \%$ tienen DM. Aún no se comprende del todo el papel que juega la DM en la etiopatogenia de la sepsis; ${ }^{18}$ empero, resultados obtenidos en múltiples estudios multicéntricos y experimentales han demostrado la fuerte asociación entre la hiperglucemia y los estados de inmunosupresión. Algunos autores sugieren que el fenómeno de entrecruzamiento molecular de los productos finales de una glucosilación avanzada tendría un efecto potencialmente inhibitorio de ciertos componentes del sistema inmunológico, lo cual repercute e incrementa la vulnerabilidad frente a procesos infecciosos y eventualmente en sepsis. Por tanto, una reducción de los niveles séricos de glucosa parece atenuar los efectos deletéreos sobre la función inmunitaria a consecuencia de la hiperglucemia. No obstante, la evidencia de una asociación causal entre hiperglucemia e infección en pacientes críticos se encuentra aún limitada y bajo investigación. ${ }^{20}$ 


\section{LA CONDICIÓN CLÍNICA Y RIESGO DE INFECCIÓN}

Shah et al, en Canadá, efectuaron el primer estudio que analizó retrospectivamente la tasa de infección y/o mortalidad en pacientes diabéticos y casos controles ajustados por grupos etarios a lo largo de dos periodos de tiempo independientes, integrando más de 500,000 casos por grupo. ${ }^{21}$ Los datos obtenidos en dicho estudio demostraron una tasa de infecciones significativamente mayor en pacientes con DM, sobre todo infecciones de etiología bacteriana como la osteomielitis, pielonefritis, neumonía, celulitis, peritonitis y sepsis. ${ }^{22}$ Además de actuar como factor de riesgo independiente para desarrollar infecciones bacterianas, hay aspectos de la DM que influyen simultáneamente en la patogénesis de la sepsis; por ejemplo, mayor susceptibilidad a infecciones por patógenos inusuales así como un mayor riesgo de experimentar complicaciones concomitantes al cuadro infeccioso. Por tanto, en pacientes diabéticos son más comunes infecciones tales como otitis externa maligna, mucormicosis cerebral y colecistitis gangrenosa, entre otras. ${ }^{23}$

En términos generales, la DM está relacionada con un riesgo incrementado de sepsis en pacientes febriles en el Servicio de Urgencias. ${ }^{20,24}$ Hay evidencia que demuestra que la colonización nasal y extranasal de Staphylococcus aureus tiene una prevalencia notable en personas con diagnóstico de DM, y que la prevalencia de sepsis por $S$. aureus en pacientes diabéticos es mayor que en los individuos sin DM. ${ }^{20,25}$ De igual forma se ha observado una elevada incidencia de DM en pacientes con sepsis por bacterias gram-negativas como Klebsiella spp. ${ }^{20}$ McKane et al fueron capaces de demostrar que un diagnóstico de DM y una hemoglobina glucosilada A mayor a 6.5\% actúan como predictores independientes de sepsis adquirida en la comunidad en la población de pacientes en estado crítico. ${ }^{20}$ Michalia et al demostraron en un estudio que, a pesar del estricto control glicémico, los pacientes diabéticos ingresados a la $\mathrm{UCl}$ tienen una probabilidad 1.7 veces mayor de desarrollar una sepsis adquirida en la $\mathrm{UCI}$ en comparación con sujetos no diabéticos y después de haber hecho ajustes según la condición clínica, edad y género. ${ }^{16}$ La susceptibilidad observada de los pacientes diabéticos en estado crítico a sepsis podría deberse parcialmente a la vasculopatía diabética, la cual podría exacerbar la isquemia relacionada a hipoperfusión y facilitar la translocación bacteriana. ${ }^{16}$ Condiciones específicas como la cetoacidosis diabética, el uso prolongado de esteroides y el uso inapropiado de un tratamiento antimicrobiano empírico se encuentran asociadas independientemente con una mortalidad temprana entre los pacientes con DM2 y sepsis adquirida en la comunidad. ${ }^{26}$ Los pacientes diabéticos con nefropatía crónica bajo tratamiento hemodialítico se encuentran en riesgo particular de desarrollar sepsis; asimismo, los factores de riesgo para morbimortalidad entre pacientes sépticos recibiendo hemodiálisis son: edad avanzada, aislamiento de Staphylococcus aureus, así como aislamiento de microorganismos resistentes. Las múltiples hospitalizaciones y la presencia de un catéter venoso central agravan aún más el escenario clínico antes descrito. ${ }^{27-29}$ Williams evidenció en un estudio que una actividad física inadecuada está relacionada con un incremento del doble en el riesgo para mortalidad por sepsis. Puesto que la incidencia de sepsis se encuentra en aumento, sus resultados sugieren que instaurar un régimen de ejercicio en las personas puede ser eficaz en reducir la incidencia de la enfermedad. ${ }^{8}$ No obstante, y de forma peculiarmente contrastante, Kuperman et al demostraron en un estudio que la obesidad podría tener un efecto protector contra la mortalidad en pacientes sépticos hospitalizados. El efecto protector de la obesidad podría tener una interdependencia con la DM, posiblemente a través de un intermediario hormonal aún no identificado. ${ }^{30}$

La sepsis es una de las causas principales de lesión renal aguda (LRA), la cual se instaura hasta en un $25 \%$ de los pacientes sépticos y en aproximadamente $50 \%$ de los pacientes con bacteremia o choque. La LRA relacionada a sepsis está asociada con tasas de mortalidad elevadas, las cuales alcanzan valores de hasta $70 \%{ }^{3}$ Es aún controversial si la DM incrementa el riesgo de LRA durante la sepsis. Sin embargo, la DM es un factor de riesgo bien establecido, tanto para LRA como para sepsis. ${ }^{3}$

\section{DIABETES MELLITUS Y EL SISTEMA INMUNOLÓGICO}

\section{Sistema del complemento}

El sistema del complemento (SC) actúa como mecanismo efector tanto de la inmunidad innata como adaptativa; está constituido por más de 30 proteínas de membrana y plasmáticas sintetizadas por los hepatocitos o de forma local en los tejidos periféricos, las cuales habitualmente circulan en forma de precursores inactivos. ${ }^{31}$ Dichas proteínas del SC interactúan entre sí dentro del contexto de tres cascadas enzimáticas: vía clásica, vía alternativa y vía de lectina de unión a manosa. Las tres vías de activación del SC convergen a nivel de los componentes C3, C5 y posteriormente comparten una secuencia de reacciones en común mediada por los componentes tardíos C6, C7, C8 y C9, generando el complejo de ataque a la membrana (CAM), el cual actúa a su vez como el principal efector del daño tisular generado por el SC. ${ }^{32}$

En los últimos años se ha acumulado evidencia que apoya la hipótesis de que la DM es una patología que interfiere con proteínas reguladoras del SC y, por lo tanto, precipita 
la activación del mismo, lo que a su vez tiene implicaciones directas en la etiopatogenia de la enfermedad, sobre todo a nivel vascular. ${ }^{33}$ Se piensa que la DM favorece la formación de ligandos activadores del SC; de hecho, estudios recientes han demostrado una estrecha asociación entre la vía de lectina de unión a manosa (LUM) de activación del SC con la nefropatía diabética; asimismo, concentraciones elevadas de LUM están asociadas con una mayor tasa de mortalidad en pacientes diabéticos. ${ }^{34}$ Por otro lado, De Vries et al fueron capaces de demostrar que la vía de LUM estaba involucrada en la activación del SC inducida por isquemia. ${ }^{33}$ Es bien sabido que la capacidad de activación del SC a través de la vía clásica se encuentra disminuida en la DM2, esto podría contribuir a la susceptibilidad aumentada a infecciones. ${ }^{35}$ Aunque algunos estudios han sido capaces de detectar una deficiencia del componente $\mathrm{C} 4$ en la DM, dicha reducción de C4 se encuentra probablemente asociada a la disfunción polimorfonuclear y a una respuesta reducida a expensas de mediadores proinflamatorios del tipo de las citocinas. ${ }^{36}$ Por otro lado, en pacientes obesos con DM2 se presenta una mayor activación de la vía alternativa del SC, la cual es favorecida aún más por las condiciones hiperquilomicrónicas postprandiales; esto induce la sobreproducción de inflamación tisular por la proteína estimuladora de acilación (PEA) y el componente C3a. Por tanto, la inflamación mediada por el SC puede contribuir de forma adicional al aceleramiento de la microangiopatía diabética. ${ }^{35}$

Ghosh et al propusieron un modelo referente a la patogenia de las complicaciones de la DM basado en la inactivación del CD59 a consecuencia de la glucosilación no enzimática condicionada por estados hiperglicémicos, lo cual suscita una activación incrementada del CAM, activación concomitante de vías de señalización intracelular, así como liberación de múltiples citocinas y factores de crecimiento con efecto proinflamatorio y protrombótico. ${ }^{37} \mathrm{Se}$ ha demostrado en experimentos in vitro que la incubación de CD59 en la presencia de azúcares reductores ocasiona una pérdida de la función reguladora del SC. Análisis preliminares también han sugerido que el CD59 aislado a partir de orina de pacientes diabéticos se encontraba glucosilado. En conjunto, dichos hallazgos llevaron a sugerir que la glucosilación de CD59 sobre células expuestas al plasma en pacientes diabéticos podría predisponer a dichas células a susceptibilidad de daño por el SC. ${ }^{38}$ Davies et al objetivaron en un estudio que la pérdida de la función del CD59 fue responsable de la susceptibilidad incrementada a la lisis y demostraron que los eritrocitos de personas diabéticas poseen poco CD59 no glucosilado activo, lo cual está en concordancia con el hallazgo usual de anemia en pacientes con DM1, así como la reticulocitosis concomitante que denota un ciclo vital acortado de los glóbulos rojos en la circulación. ${ }^{38}$
Por otro lado, varios estudios sugieren una asociación entre el componente C3 del complemento con un estado de hipofibrinólisis en pacientes con DM1, lo anterior debido a un incremento en los niveles plasmáticos de inhibidor del activador de plasminógeno-1 (IAP-1), lo cual tendría repercusiones significativas en la etiopatogenia de las complicaciones de la DM, sobre todo las de carácter trombótico a nivel cardiovascular. ${ }^{39}$

\section{Monocitos y linfocitos}

En el contexto de la DM, los monocitos no han sido tan estudiados como los granulocitos neutrófilos; no obstante, es bien sabido que presentan alteraciones en la quimiotaxis y la fagocitosis. ${ }^{40}$ En años recientes se ha estudiado la regulación que ejerce la vía de señalización Hedgehog $(\mathrm{Hh})$ como potente quimioatrayente de los monocitos, lo cual se ha corroborado en DM. ${ }^{41}$ En contraste, Nandy et al demostraron que la hiperglucemia favorecía la activación monocítica puesto que fenómenos como la adhesión, migración y transmigración de dichas células inmunitarias se encontraban aumentados. ${ }^{42}$ Casqueiro et al encontraron que las células mononucleares y los monocitos de pacientes con DM secretan menos interleucina-1 (IL-1) e interleucina-6 (IL-6) en respuesta a la estimulación con lipopolisacáridos de bacterias gram-negativas. ${ }^{36}$

Por el momento existen pocos estudios referentes al efecto de la DM sobre la función linfocitaria. Algunos estudios han reportado que la glucosilación proteica incrementada podría inhibir la producción de interleucina-10 (IL-10) a partir de células mieloides, así como la producción de Interferón- $\gamma($ IFN- $\gamma$ ) y factor de necrosis tumoral- $\alpha$ (FNT- $\alpha$ ) por los linfocitos-T. ${ }^{36}$ En contraste, se ha descubierto que existe un efecto negativo del FNT- $\alpha$ y la IL-1 en el desarrollo y la función de los linfocitos-T reguladores; por tanto, inhibir dichas citocinas podría tener efectos benéficos en la regulación inmunológica en pacientes con DM1. ${ }^{43}$ Existe evidencia que apoya la hipótesis de que la glucosilación proteica avanzada podría reducir la expresión del complejo mayor de histocompatibilidad $(\mathrm{CMH})$ clase I en la superficie de las células mieloides, alterando la inmunidad celular. ${ }^{36}$ Restrepo et al demostraron en un estudio que la fagocitosis mediada por el SC o receptores $\mathrm{FC}_{\mathrm{C}} \boldsymbol{\gamma}$ se encuentra alterada en los monocitos de pacientes con DM2 con hiperglucemia crónica. ${ }^{44} \mathrm{El}$ factor nuclear- $\kappa \mathrm{B}(\mathrm{FN}-\kappa b)$ y la proteína activadora-1 (PA-1) son factores de transcripción inducidos por la hiperglucemia. La activación de dichos factores de transcripción modula los procesos de inflamación, disfunción endotelial, agregación plaquetaria y apoptosis. ${ }^{20}$ Por tanto, el $\mathrm{FN}-\kappa \mathrm{B}$ es un factor de transcripción nuclear inducible que juega un papel central en la regulación de la transcripción de diversos genes, incluyendo aquellos 
que codifican las citocinas inflamatorias, involucradas en la sepsis severa. ${ }^{7}$ La hiperglucemia también incrementa la generación de especies reactivas de oxígeno, ocasionando daño tisular directo y reduciendo la perfusión tisular. ${ }^{20}$

\section{Granulocitos neutrófilos}

Se ha demostrado que los granulocitos neutrófilos de pacientes diabéticos presentan múltiples defectos funcionales; existe producción excesiva de citocinas y enzimas las cuales, aunadas a un estado inflamatorio prolongado, pueden contribuir a la activación vascular inapropiada y daño tisular. ${ }^{45}$

El reclutamiento de los granulocitos neutrófilos al sitio inflamatorio precisa de fenómenos de adhesión endotelial y transmigración, los cuales a su vez requieren de la interacción entre integrinas leucocitarias y diversas moléculas de adhesión celular expresadas en la superficie de las células endoteliales. ${ }^{46}$ Actualmente existe evidencia de una expresión incrementada de integrinas y moléculas de adhesión celular ante situaciones de hiperglucemia. Basta et al demostraron que los productos finales de la glucosilación avanzada favorecían la expresión de molécula de adhesión celular vascular-1 (MACV-1), molécula de adhesión intercelular-1 (MAIC-1) y selectina-E, las cuales incrementan la adhesión leucocitaria a células endoteliales. ${ }^{47}$ Umsa-ard et al demostraron en un estudio con granulocitos neutrófilos incubados en un medio rico en glucosa que dichas células presentaban mayor migración hacia sitios con elevada concentración de interleucina-8 (IL-8) y una mejor adherencia a células endoteliales. ${ }^{48}$ Spiller et al demostraron experimentalmente en ratones diabéticos una mayor susceptibilidad a sepsis polimicrobiana debido a una reducción en los procesos leucocitarios de rodamiento, adhesión y migración al foco de infección; igualmente demostraron que la expresión de CXCR2 en granulocitos neutrófilos estaba considerablemente reducida en ratones diabéticos, en comparación con ratones no diabéticos. Por último, se pudo evidenciar que la expresión del ácido ribonucleico mensajero (ARNm) hepático de la glucoproteína ácida- $\alpha 1$ (GPA) estaba aumentada en ratones en estado séptico; dicha proteína alteraba la migración de los granulocitos neutrófilos al foco infeccioso. ${ }^{6}$

Se ha demostrado experimentalmente que en condiciones de hiperglucemia se incrementa la expresión de moléculas de adhesión en la superficie de los granulocitos neutrófilos, tal es el caso de CD11b, la cual favorece su adhesión espontánea a células endoteliales. ${ }^{23}$ En contraste, la hiperglucemia altera la quimiotaxis neutrofílica y ocasiona efectos deletéreos en las actividades bactericidas como la fagocitosis. Asimismo, dichas condiciones hiperglicémicas crónicas alteran la actividad bactericida al inhibir la enzima glucosa-6-fosfato deshidrogenasa (G6FD), lo cual incrementa la apoptosis de los leucocitos polimorfonucleares $y$, por tanto, se reduce la migración transendotelial de dichas células. ${ }^{36}$ Savu et al encontraron en un estudio que la actividad plasmática de la enzima granulocítica mieloperoxidasa (MPO) se encuentra incrementada en pacientes diabéticos en comparación con los no diabéticos, asimismo la actividad MPO está directamente relacionada con los valores de Hb1Ac. Lo anterior sugiere que la DM está caracterizada por un estado de inflamación crónica. ${ }^{49,50}$

Se sabe que los granulocitos neutrófilos liberan enzimas y cromatina al ser activados, originando las denominadas trampas extracelulares de neutrófilos (TEN), las cuales confieren un mecanismo defensivo natural frente a la respuesta inflamatoria; no obstante, una liberación excesiva de dichas sustancias genera efectos protrombóticos y proinflamatorios con la disfunción endotelial concomitante (Figura 1). ${ }^{51}$ Diversos autores han propuesto una interrelación entre la hiperglucemia y el estrés oxidativo inducido por actividad incrementada de los neutrófilos, la cual tendría implicaciones directas en la patogenia de las complicaciones de la DM. ${ }^{52}$

\section{Marcadores inflamatorios}

Los niveles de citocinas proinflamatorias y quimiocinas circulantes como por ejemplo el FNT- $\alpha$, interleucina- $1 \beta$ (IL-1 $\beta)$, y la proteína quimioatrayente de monocitos-1 (PQM-1) son utilizados comúnmente como marcadores de la inflamación y se han demostrado incrementados en la DM. ${ }^{53,54}$ Se ha demostrado que la resistencia a la insulina está atribuida parcialmente a niveles plasmáticos elevados de citocinas proinflamatorias. Por otro lado, Yende et al demostraron que la DM constituía un factor de riesgo para un mal pronóstico en pacientes con neumonía; sin embargo, no fueron capaces de evidenciar diferencias consistentes en la respuesta inflamatoria temprana pues existía una liberación normal de citocinas tales como IL-10, IL-6 y FNT- $\alpha .^{23,55}$

Actualmente, los factores relacionados a la inflamación son la proteína-C reactiva ultrasensible (PCR-US), el receptor tipo toll-2 (RTT2), el receptor tipo toll-4 (RTT4), el IAP-1, IL-1 $\beta$, IL-6, FNT- $\alpha .{ }^{56}$ Otros mediadores proinflamatorios son: FNT- $\alpha$, PCR-US y PQM-1, los cuales podrían tener un papel independiente en varias vías de señalización del estrés oxidativo relacionado a hiperglucemia. Un incremento en el estrés oxidativo podría amplificar aún más la respuesta inflamatoria, estableciendo un ciclo vicioso. ${ }^{57}$ Niveles séricos elevados de PQM-1 podrían contribuir al inicio y progresión de distintas complicaciones de la DM. Por tanto, la PQM-1 sérica podría actuar como biomarcador de actividad inflamatoria y auxiliar en la detección precoz e intervención precisa de complicaciones diabéticas. ${ }^{58}$ 


\section{DIABETES MELLITUS Y LA RESPUESTA INMUNOLÓGICA EN SEPSIS}

Investigaciones previas han sugerido que los pacientes diabéticos están particularmente predispuestos a disfunción endotelial durante la sepsis, ya que la hipergluce- mia, resistencia a la insulina y obesidad activan distintas vías endoteliales aun en condiciones no sépticas. ${ }^{18,59}$ La evidencia emergente sugiere que la activación y disfunción celular endotelial juegan un papel esencial en la fisiopatología de la sepsis; asimismo el endotelio es crítico en la fuga vascular y el estado de choque

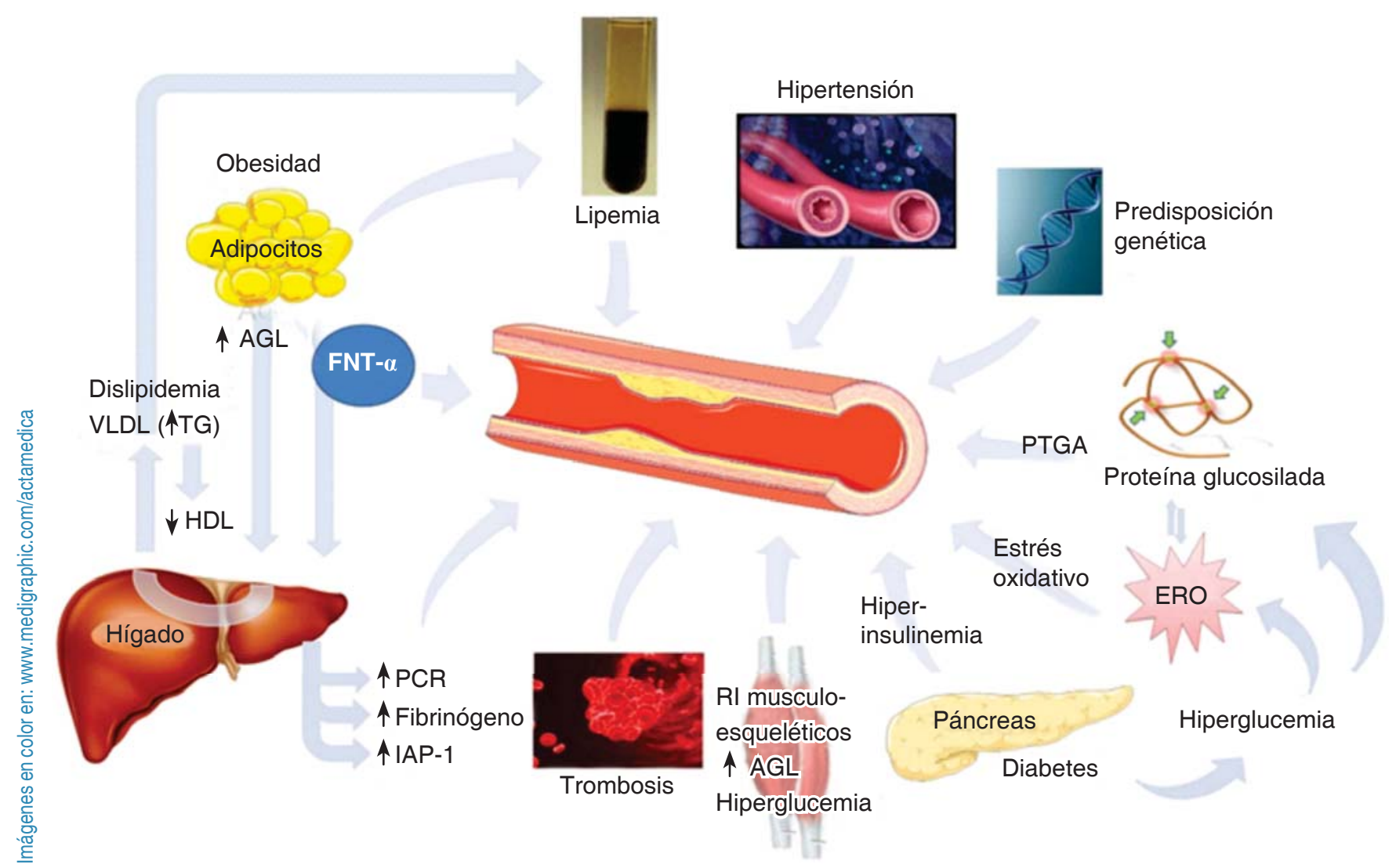

Figura 1. Diversos mecanismos que fomentan la disfunción endotelial y el daño vascular en la DM2. Varios factores de riesgo convergen en la arteria (centro) para promover la aterogénesis bajo condiciones diabéticas. Dichos factores incluyen: hipertensión, predisposición genética, hiperglucemia, hiperinsulinemia, estrés oxidativo, productos terminales de la glucosilación avanzada (PTGA), resistencia a la insulina y ácidos grasos libres (AGL) circulantes incrementados, lipemia, obesidad incrementada en relación a factores que caracterizan un estilo de vida (sedentarismo, etilismo, tabaquismo y hábitos alimenticios), citocinas proinflamatorias y protrombóticas incrementadas. Los tejidos periféricos son resistentes a la acción de la insulina, lo cual promueve la hiperglucemia y niveles incrementados de AGL. En estados de resistencia a la insulina, el páncreas inicialmente trata de compensar mediante la mayor producción de insulina, resultando en hiperinsulinemia, un factor de riesgo en sí mismo para angiopatía. Los niveles elevados de grasa abdominal presentan al hígado con niveles elevados de AGL a través de la circulación portal. Dicho exceso de AGL conduce a un exceso de producción de partículas de lipoproteína ricas en triglicéridos (TG). La hipertrigliceridemia se encuentra acompañada por una disminución concomitante en el colesterol HDL. El adipocito también es capaz de liberar citocinas proinflamatorias como el FNT- $\alpha$, las cuales no solamente tienen efectos directos en la pared vascular al promover la aterogénesis, sino también pueden provocar la producción de reactantes de fase aguda en el hígado, incluyendo la PCR, fibrinógeno e IAP-1. Finalmente, la formación de PTGA a partir de macromoléculas glucosiladas puede dañar la vasculatura a través de distintos mecanismos. HDL, high-density lipoprotein (lipoproteína de alta densidad); VLDL, very low-density lipoprotein (lipoproteína de muy baja densidad); FNT- $\alpha$, factor de necrosis tumoral- $\alpha$ PCR, proteína C-reactiva; IAP-1, inhibidor del activador del plasminógeno-1; ERO, especies reactivas de oxígeno.

Traducido y adaptado con permiso de Elsevier: Sena CM, Pereira AM, Seiça R. Endothelial dysfunction-A major mediator of diabetic vascular disease. Biochim Biophys Acta. 2013; 1832 (12): 2216-2231. Derechos Reservados. 
subsecuente que contribuyen a la mortalidad entre los pacientes sépticos. ${ }^{18}$

Estudios clínicos han demostrado niveles incrementados de los marcadores de adhesión selectina-E, MAIC-1 y MACV-1 en pacientes con DM. También se han encontrado niveles incrementados de IAP-1 en pacientes con DM, lo cual incrementa su riesgo para enfermedad trombótica. ${ }^{18}$ Schuetz et al demostraron niveles incrementados de selectina-E y sFLT-1, un receptor del factor de crecimiento endotelial vascular (FCEV), en pacientes diabéticos durante las etapas más severas de la sepsis, específicamente el choque séptico, en comparación con pacientes no diabéticos. ${ }^{18}$ Hay información antagónica como la de Filgueiras et al que demostraron que durante la sepsis los pacientes diabéticos tienen menor probabilidad de desarrollar insuficiencia respiratoria aguda (IRA) e inclusive síndrome de dificultad respiratoria aguda (SDRA) en comparación con pacientes no diabéticos. ${ }^{60}$ Este fenómeno podría estar relacionado con una activación disminuida de $\mathrm{FN}-\kappa \mathrm{B}$ en macrófagos alveolares, el cual se considera un mecanismo molecular que provee protección pulmonar en pacientes diabéticos con sepsis. ${ }^{60}$ Zhao y colaboradores demostraron en un estudio que tanto C3, el CAM y la LUM estaban significativamente elevadas en pacientes sépticos con coagulación intravascular diseminada (CID). El CAM fue un útil predictor de CID inducida por sepsis. Otros resultados son ambiguos, como el de Chang et al donde los pacientes diabéticos con sepsis severa que requirieron internamiento en la $\mathrm{UCI}$ no tuvieron peor evolución clínica que los pacientes no diabéticos. ${ }^{17}$

\section{CONCLUSIONES}

La DM, debido a su gran prevalencia y potencial para alterar elementos fundamentales en la fisiopatología de la sepsis, se considera una condición comórbida; sin embargo, aún no se conoce a plenitud la influencia que ejerce la DM en la infección y en el desarrollo de la sepsis.

La DM se considera como un estado de inmunosupresión y en el caso particular de los pacientes sépticos, éstos se encuentran susceptibles a una disfunción endotelial, lo cual se piensa que pueda repercutir en la evolución del cuadro clínico. La DM se asocia a una susceptibilidad incrementada a enfermedades infecciosas y la evidencia epidemiológica que avale esta asociación es suficiente. Sin embargo, no está claro si la DM influye sustancialmente en el curso clínico y/o pronóstico de un proceso infeccioso. La mayor parte de los estudios han evidenciado un déficit funcional de los granulocitos neutrófilos, anormalidades en los fenómenos de adhesión, quimiotaxis y fagocitosis y destrucción intracelular de microorganismos patógenos.
No obstante, no se comprende enteramente si los trastornos metabólicos propios de la DM explican el nexo con la susceptibilidad a procesos infecciosos.

Aparte del defecto en la inmunidad celular, actualmente hay evidencia indicativa de una respuesta inmunitaria humoral precaria, reflejada en una menor producción de anticuerpos y componentes del SC con efecto de opsonización, lo cual podría influir en la patogenia de las infecciones. Mecanismos moleculares como la modificación de proteínas, efectos osmóticos en el huésped también han sido propuestos sin evidencia contundente.

La hiperglucemia está asociada con tasas de mortalidad elevadas, por lo que mantener un estado de normoglucemia con el empleo de infusiones continuas de insulina mejora el desenlace clínico en pacientes en estado crítico.

Finalmente, la carga socioeconómica generada por la DM es significativa e incrementa de manera progresiva. En términos financieros, su impacto es cada vez mayor, puesto que se estima que la proporción de individuos con DM aumentará en los próximos años y también se espera que su impacto en la etiopatogenia de complicaciones de carácter infeccioso sea superior, por ello resultaría oportuno prevenir las infecciones en dicho grupo de población, al mismo tiempo que se reduciría la carga económica para los sistemas de salud.

\section{REFERENCIAS}

1. Lontchi-Yimagou E, Sobngwi E, Matsha TE, Kengne AP. Diabetes mellitus and inflammation. Curr Diab Rep. 2013; 13: 435-444.

2. Rhodes ET, Prosser LA, Hoerger TJ, Lieu T, Ludwig DS, Laffel LM. Estimated morbidity and mortality in adolescents and young adults diagnosed with type 2 diabetes mellitus. Diabet Med. 2012; 29 (4): 453-463.

3. Venot M, Weis L, Clec'h C, Darmon M, Allaouchiche B, GoldgranTolédano D et al. Acute kidney injury in severe sepsis and septic shock in patients with and without diabetes mellitus: a multicenter study. PLoS One. 10(5): e0127411. doi:10.1371/journal.pone.0127411.

4. Guariguata L, Whiting DR, Hambleton I, Beagley J, Linnenkamp U, Shaw JE. Global estimates of diabetes prevalence for 2013 and projections for 2035. Diabetes Res Clin Pr. 2014; 103 (2): 137-149.

5. Liberty IF, Freha NA, Baumfeld Y, Codish S, Schlaeffer F, Novack V. Prognostic value of glycated hemoglobin for one year mortality following hospitalization in the internal medicine ward. Isr Med Assoc J. 2015; 17: 277-281.

6. Spiller F, Carlos D, Souto FO, de Freitas A, Soares FS, Vieira SM et al. $\alpha 1$-acid glycoprotein decreases neutrophil migration and increases susceptibility to sepsis in diabetic mice. Diabetes. 2012; 61: 1584-1591.

7. Uyanik A, Unal D, Uyanik MH, Halici Z, Odabasoglu F, Altunkaynak $\mathrm{ZB}$ et al. The effects of polymicrobial sepsis with diabetes mellitus on kidney tissues in ovariectomized rats. Ren Fail. 2010; 32: 592-602.

8. Williams PT. Inadequate exercise as a risk factor for sepsis mortality. PLoS One. 8 (12): e79344. doi:10.1371/journal.pone.0079344.

9. Korbel L, Spencer JD. Diabetes mellitus and infection: an evaluation of hospital utilization and management costs in the United States. J Diabetes Complicat. 2015; 29: 192-195.

10. Finfer S. Clinical controversies in the management of critically ill patients with severe sepsis: resuscitation fluids and glucose control. Virulence. 2014; 5 (1): 183-188. 
11. McDonald HI, Nitsch D, Millett ERC, Sinclair A, Thomas SL. New estimates of the burden of acute community-acquired infections among older people with diabetes mellitus: a retrospective cohort study using linked electronic health records. Diabet Med. 2014; 31 : 606-614.

12. Edwards R, Hutson R, Johnson J, Sherwin R, Gordon-Strachan G, Frankson $M$ et al. Severe sepsis in the emergency department - an observational cohort study from the university hospital of the west indies. West Indian Med J. 2013; 62 (3): 224-229.

13. Kumar G, Kumar N, Taneja A, Kaleekal T, Tarima S, McGinley E et al. Nationwide trends of severe sepsis in the 21st century (2000-2007). Chest. 2011; 140: 1223-1231.

14. Boomer JS, To K, Chang KC, Takasu O, Osborne DF, Walton AH et al. Immunosuppression in patients who die of sepsis and multiple organ failure. J Am Med Assoc. 2011; 306: 2594-2605.

15. Quan HB, Li TY, Gao YY, Chen DX. Clinical features of diabetes mellitus cases complicated by Burkholderia pseudomallei septicemia. Genet Mol Res. 2014; 13 (2): 3108-3116.

16. Michalia M, Kompoti M, Koutsikou A, Paridou A, Giannopoulou P, Trikka-Graphakos E et al. Diabetes mellitus is an independent risk factor for ICU-acquired bloodstream infections. Intensive Care Med. 2009; 35: 448-454.

17. Chang CW, Kok VC, Tseng TC, Horng JT, Liu CE. Diabetic patients with severe sepsis admitted to intensive care unit do not fare worse than non-diabetic patients: a nationwide population-based cohort study. PLoS One. 2012; 7 (12): e50729. doi:10.1371/journal. pone.0050729.

18. Schuetz P, Yano K, Sorasaki M, Ngo L, St Hilaire M, Lucas JM et al. Influence of diabetes on endothelial cell response during sepsis. Diabetologia. 2011; 54: 996-1003.

19. Singh SK, Sridhar GR. Infections and diabetes. Int J Diabetes Dev Ctries. 2015; 35 (2): 59-62.

20. McKane CK, Marmarelis M, Mendu ML, Moromizato T, Gibbons FK, Christopher KB. Diabetes mellitus and community-acquired bloodstream infections in the critically ill. J Crit Care. 2014; 29: 70-76.

21. Shah BR, Hux JE. Quantifying the risk of infectious diseases for people with diabetes. Diabetes Care. 2003; 26: 510-513.

22. Seshasai SR, Kaptoge S, Thompson A, Di Angelantonio E, Gao P, Sarwar $\mathrm{N}$ et al. Diabetes mellitus, fasting glucose, and risk of causespecific death. N Engl J Med. 2011; 364: 829-841.

23. Knapp S. Diabetes and infection: is there a link? - a mini-review. Gerontology 2013; 59: 99-104.

24. Vincent JL, Rello J, Marshall J, Silva E, Anzueto A, Martin CD et al. International study of the prevalence and outcomes of infection in intensive care units. JAMA. 2009; 302: 2323-2329.

25. Khan MAA, Hunter JM, Tan C, Seleem M, Stride PJO. Staphylococcal sepsis with multiple abscesses, urinary tract infection, and bilateral renal vein thrombosis in a patient with uncontrolled diabetes mellitus. Case Report Med. 2012; 2012: 357502. doi:10.1155/2012/357502.

26. Yo CH, Lee MTG, Gi WT, Chang SS, Tsai KC, Chen SC et al. Prognostic determinants of community-acquired bloodstream infection in type 2 diabetic patients in ED. Am J Emerg Med. 2014; 32: 1450-1454.

27. Fram D, Okuno MF, Taminato M, Ponzio V, Manfredi SR, Grothe C. Risk factors for bloodstream infection in patients at a Brazilian hemodialysis center: a case-control study. BMC Infect Dis. 2015; 15: 158.

28. Fram D, Taminato M, Ponzio V, Manfredi SR, Grothe C, Batista RE. Risk factors for morbidity and mortality of bloodstream infection in patients undergoing hemodialysis: a nested case-control study. BMC Res Notes. 2014; 7: 882.

29. Khan FG, Ahmed E. Acute renal failure in diabetes mellitus. J Pak Med Assoc. 2015; 65: 179-182.

30. Kuperman EF, Showalter JW, Lehman EB, Leib AE, Kraschnewski JL. The impact of obesity on sepsis mortality: a retrospective review. BMC Infect Dis. 2013; 13: 377.
31. Mellbin LG, Bjerre M, Thiel S, Hansen TK. Complement activation and prognosis in patients with type 2 diabetes and myocardial infarction. Diabetes Care. 2012; 35: 911-917.

32. Mathern DR, Heeger PS. Molecules great and small: the complement system. Clin J Am Soc Nephrol. 2015; 10(9): 1636-1650.

33. Geng P, Ding Y, Qiu L, Lu Y. Serum mannose-binding lectin is a strong biomarker of diabetic retinopathy in chinese patients with diabetes. Diabetes Care. 2015; 38 (5): 868-875.

34. Østergaard JA, Bjerre M, Dagnaes-Hansen F, Hansen TK, Thiel S, Flyvbjerg A. Diabetes-induced changes in mannan-binding lectin levels and complement activation in a mouse model of type 1 diabetes. Scand J Immunol. 2013; 77: 187-194.

35. Fujita T, Hemmi S, Kajiwara M, Yabuki M, Fuke Y, Satomura A et al. Complement-mediated chronic inflammation is associated with diabetic microvascular complication. Diabetes Metab Res Rev. 2013; 29: 220-226.

36. Casqueiro J, Casqueiro J, Alves C. Infections in patients with diabetes mellitus: A review of pathogenesis. Indian J Endocrinol Metab. 2012; 16: S27-36.

37. Ghosh P, Vaidya A, Sahoo R, Goldfine A, Herring N, Bry L et al. Glycation of the complement regulatory protein CD59 is a novel biomarker for glucose handling in humans. J Clin Endocrinol Metab. 2014; 99 (6): 999-1006.

38. Davies CS, Harris CL, Morgan BP. Glycation of CD59 impairs complement regulation on erythrocytes from diabetic subjects. Immunology. 2005; 114: 280-286.

39. Hess K, Alzahrani SH, Price JF, Strachan MW, Oxley N, King R et al. Hypofibrinolysis in type 2 diabetes: the role of the inflammatory pathway and complement C3. Diabetologia. 2014; 57: 1737-1741.

40. Yang M, Gan H, Shen Q, Tang W, Du X, Chen D. Proinflammatory CD14+CD16+ monocytes are associated with microinflammation in patients with type 2 diabetes mellitus and diabetic nephropathy uremia. Inflammation. 2012; 35 (1): 388-396.

41. Dunaeva M, Voo S, van Oosterhoud C, Waltenberger J. Sonic hedgehog is a potent chemoattractant for human monocytes: diabetes mellitus inhibits Sonic hedgehog-induced monocyte chemotaxis. Basic Res Cardiol. 2010; 105: 61-71.

42. Nandy D, Janardhanan R, Mukhopadhyay D, Basu A. Effect of hyperglycemia on human monocyte activation. J Investig Med. 2011; 59 (4): 661-667.

43. Thomas HE, Graham KL, Chee J, Thomas R, Kay TW, Krishnamurthy B. Proinflammatory cytokines contribute to development and function of regulatory T cells in type 1 diabetes. Ann N Y Acad Sci. 2013; 1283: 81-86.

44. Restrepo BI, Twahirwa M, Rahbar MH, Schlesinger LS. Phagocytosis via complement or fc-gamma receptors is compromised in monocytes from type 2 diabetes patients with chronic hyperglycemia. PLoS One. 2014; 9 (3): e92977. doi:10.1371/journal.pone.0092977.

45. Amulic B, Cazalet C, Hayes GL, Metzler KD, Zychlinsky A. Neutrophil function: from mechanisms to disease. Annu Rev Immunol. 2012; 30: 459-489.

46. Kolaczkowska E, Kubes P. Neutrophil recruitment and function in health and inflammation. Nat Rev Immunol. 2013; 13: 159-175.

47. Basta G, Lazzerini G, Del Turco S, Ratto GM, Schmidt AM, De Caterina R. At least 2 distinct pathways generating reactive oxygen species mediate vascular cell adhesion molecule- 1 induction by advanced glycation end products. Arterioscler Thromb Vasc Biol. 2005; 25 (7): 1401-1407.

48. Umsa-ard W, Thongboonkerd V, Soongsathitanon J. Activated status and altered functions of neutrophils in poorly controlled diabetes. J ASEAN Fed Endocr Soc. 2015; 30 (1): 9-17.

49. Savu O, Serafinceanu C, Grajdeanu IV, Iosif L, Gaman L, Stoian I. Paraoxonase lactonase activity, inflammation and antioxidant status in plasma of patients with type 1 diabetes mellitus. J Int Med Res. 2014; 42 (2): 523-529. 
50. Gorudko IV, Kostevich VA, Sokolov AV, Shamova EV, Buko IV, Konstantinova EE. Functional activity of neutrophils in diabetes mellitus and coronary heart disease: role of myeloperoxidase in the development of oxidative stress. Exp Biol Med. 2012; 154 (7): 28-36.

51. Menegazzo L, Ciciliot S, Poncina N, Mazzucato M, Persano M, Bonora $B$ et al. NETosis is induced by high glucose and associated with type 2 diabetes. Acta Diabetol. 2015; 52: 497-503.

52. Almyroudis NG, Grimm MJ, Davidson BA, Röhm M, Urban CF, Segal $\mathrm{BH}$. NETosis and NADPH oxidase: at the intersection of host defense, inflammation, and injury. Front Immunol. 2013; 4: 45. doi:10.3389/ fimmu.2013.00045.

53. Min D, Brooks B, Wong J, Salomon R, Bao W, Harrisburg B et al. Alterations in monocyte CD16 in Association with Diabetes Complications. Mediat Inflamm. 2012; 2012 649083: 10. doi:10.1155/2012/649083.

54. Dalmas E, Venteclef N, Caer C, Poitou C, Cremer I, Aron-Wisnewsky J et al. T cell-derived IL-22 amplifies IL-1b-driven inflammation in human adipose tissue: relevance to obesity and type 2 diabetes. Diabetes. 2014; 63: 1966-1977.

55. Yende $S$, van der Poll T, Lee $M$, Huang DT, Newman AB, Kong L. The influence of pre-existing diabetes mellitus on the host immune response and outcome of pneumonia: analysis of two multicentre cohort studies. Thorax. 2010; 65 (10): 870-877.

56. Zhang M, Chen P, Chen S, Sun Q, Zeng QC, Chen JY et al. The association of new inflammatory markers with type 2 diabetes mellitus and macrovascular complications: a preliminary study. Eur Rev Med Pharmacol Sci. 2014; 18: 1567-1572.

57. Gupta S, Gambhir JK, Kalra OP, Gautam A, Shukla K, Mehndiratta M et al. Association of biomarkers of inflammation and oxidative stress with the risk of chronic kidney disease in Type 2 diabetes mellitus in North Indian population. J Diabetes Complications. 2013; 27: 548-552.

58. Radhakrishnan P, Srikanth P, Seshadri KG, Barani R, Samanta M. Serum monocyte chemoattractant protein-1 is a biomarker in patients with diabetes and periodontitis. Indian J Endocr Metab. 2014; 18: 505-510.

59. Schuetz P, Castro P, Shapiro NI. Diabetes and sepsis: preclinical findings and clinical relevance. Diabetes Care. 2011; 34 (3): 771-778.

60. Filgueiras LR, Martins JO, Serezani CH, Capelozzi VL, Montes MBA, Jancar S. Sepsis-induced acute lung injury (ALI) is milder in diabetic rats and correlates with impaired NF-אB activation. PLoS One. 2012; 7 (9): e44987. doi:10.1371/journal.pone.0044987. 